\title{
INVESTIGAÇÃO DA BRUCELOSE EM BOVINOS E EM CONSUMIDORES HUMANOS DO LEITE *
}

\author{
Ayrton Pinheiro de Souza** \\ Djalma de Carvalho Moreira Filho*** \\ Manildo Fávero ****
}

\begin{tabular}{l|l}
\cline { 2 - 2 } & RSPU-B/355 \\
\hline
\end{tabular}

Souza, A. P. de et al. Investigação da brucelose em bovinos e em consumidores humanos do leite. Rev. Saúde públ., S. Paulo $11: 238-47,1977$.

Resumo: O uso do leite "in natura" por certos consumidores, tem se constituido num sério problema de Saude Pablica. Desta forma, foi efetuado um levantamento da situação dos rebanhos bovinos na regiāo de Ribeirão Preto, $S P$ (Brasil) para se verificar a prevalência de brucelose. A partir da deteç̧ão de um rebanho leiteiro brucélico foram investigados os consumidores humanos do leite suspeito, segundo algumas caracteristicas, tais como: sexo, cor, idade, ocupação, estado civil, tempo de residencia na área, tempo de uso do leite suspeito, tipo de leite ingerido, quantidade de ingestão diária do leite, condições de saúde, uso de medicamentos e morbidades referidas na época do estudo. Foram feitas provas sorológicas para diagnóstico de brucelose no rebanho leiteiro e na populaçăo humana consumidora do leite. Observou-se aue cerca de 8\%o das vacas em lactaçäo na época do levantamento foram consideradas britcélicas. Entre os consumidores nào se observou casos de sorologia positiva para brucelose. Concluill-se que: apesar da importância da brucelose como zoonose, ela continua endêmica no nosso meio criatório; a fervura do loite é uma medida eficaz e deve ser preconizada nas regióes onde o leite c consumido "in natura", como medida de escolha em saide piblica.

UNITERMos: Bracelose. Brucelose bovina. Leite "in natura".

T N TRODL CA O

Das zoonoses que afetam o homen, a brucelose é uma das mais disseminadas. $O$ número de infecções humanas que ocorrem em todo o mundo é da ordem de centenas de mithares ".
A brucelose pode ser transmitida direta ou indiretamente do animal ao homem e do ponto de vista da Saúde Pública deve ser considerada não só como causa de enfermidade, de incapacidade para o trabalho e

\footnotetext{
* Trabalho realizado no Departamento de Medicina Social da Faculdade de Medicina de Ribeirão Preto da USP - Ribeirão Preto, SP.

** Ex-Médico Veterinário da Secretaria da Agricultura do Estado de São Paulo - Casa da Agricultura de Ribeirão Preto - In memorian.

*** Do Departamento de Medicina Preventiva e Social da Faculdade de Ciências Médicas da UNICAMP - Campinas SP - Brasil e do Departamento de Medicina Social da Faculdade de Medicina de Ribeiráa Preto da USP - Rua Bernardino de Campos, 1000 — Ribeirão Preto, SP - Brasil.

**** Do Departamento de Medicina Preventira e Social da Faculdade de Ciências Médicas da UNICAMP - Campinas, SP - Brasil.
} 
SouzA, A. P. de et al, - Investigação da bruce lose em bovinos e em consumidores humanos do leite, Rev. Saúde públ., S. Paulo, 11:238-47 1977.

de diminuição do rendimento, mas também como fator nocivo para a produção de alimentos, principalmente de proteinas de origem animal que são indispensáveis para a saúde e bem estar ${ }^{5}$.

$\mathrm{Na}$ vaca, a brucelose provoca redução da produção leiteira entre 20 a $25 \%$; abortos de 20 a $30 \%$; mortalidade de bezerros (de 0 a 12 meses) de 20 a $25 \%$; esterilidade de 10 a $20 \%$ e perda de peso de 10 a $15 \%{ }^{10}$.

Como a brucelose não se transmite habitualmente de um ser humano a outro, a profilaxia no homem se atem ao combate e à eliminação da doença nos animais. Uma vaca brucélica pode eliminar quantidades de Brucellas suficientes para contaminar todo o rebanho de uma região, seja através das membranas fetais, dos corrimentos puerperais ou do leite ${ }^{10}$. Os meios de contaminação mais freqüentes para o homem são: (a) os produtos alimentícios preparados do leite cru de animais infectados; (b) legumes crus contaminados por excrementos de animais infectados; (c) as víceras, medula espinhal e gânglios linfáticos de carnes infectadas, nas quais a Brucella pode permanecer viável por mais de um mês após o abate, e mais tempo ainda se congelada ou refrigeradas; e (d) a água de cisternas e poços contaminados por excrementos de animais doentes 6,5 .

Todavia, graças ao controle efetivo da doença em animais, principalmente entre os bovinos, nos EUA, por exemplo, foi possivel reduzir a brucelose humana de 6.321 casos em 1947 para 231 em 1969 4. Neste mesmo periodo o número de reatores bovinos declinou de $5 \%$ para menos de $0,1 \%{ }^{11}$.

A brucelose tanto pelo número e enfermos que causa, como pelas importantes perdas econômicas que provoca, constitui um $_{*}$ grave motivo de preocupação. No Estado de São Paulo, por exemplo, as perdas econômicas causadas pela brucelose no rebanho bovino de 1965 a 1967 foram da monta de US $\$ 158.407,00^{13}$.

A transmissão de Brucella ao homem pela ingestão e/ou manipulação do leite conta- minado, e seus derivados, está bem comprovada, e sabe-se que as três espécies principais de Brucella ( $B$. abortus; $B$. suis e $B$. melitensis) podem ser transmitidas pelo leite $6,5,14$.

O objetivo deste trabalho é investigar a presença de brucelose em um rebanho leiteiro e a possível ocorrência da doença em consumidores do leite "in natura" proveniente deste rebanho.

\section{MATERIAL E METODOS}

No início do segundo semestre de 1975 foi tomado conhecimento da possível existência de brucelose num rebanho bovino de uma propriedade rural localizada no município de Dumont, Estado de São Paulo. Soube-se, também, que o leite proveniente desse rebanho vinha sendo fornecido a um grupo de famílias do município, sendo que parte dele era também vendido a usinas de industrialização de leite.

Em função desta suspeita foi proposto um levantamento sorológico do gado para imunodiagnóstico de brucelose pelo método de soroaglutinação rápida em câmara de Huddleson 9. Este teste foi o escolhido dada as suas características de exequibilidade em campo.

Foi colhido sangue de todos animais com mais de um ano de vida que pertenciam ao rebanho, confirmando-se elevada percentagem de positividade, inclusive entre as vacas em lactação.

Diante deste fato foi planejado um levantamento dentre os consumidores do leite com o objetivo de se detectar possiveis infecções por Brucella.

Neste sentido, decidiu-se coletar informações sobre as características das pessoas expostas ao risco, tais como: idade, sexo, cor, estado civil, ocupação (profissāo) e naturalidade, que pudessem ser relacionadas à possivel ocorrência da doença.

Em vista da contaminação poder ter ocorrido em outra oportunidade, foi decidido que 
SoUZA, A. P. de et al. - Investigação da brucelose em bovinos e em consumidores humanos do lète, Rev. Saúde públ., S. Paulo, 11:238-47 1977.

se levantaria o tempo de residência no município, bem como a residência anterior, com o objetivo de se detectar possiveis focos da contaminação em outras regióes.

A fonte de fornecimento de leite a uma família, geralmente não é única, por isso se decidiu investigar os diferentes tipos de leite usados.

É sabidamente conhecido que a pasteurização e mesmo a fervura adequada previne a infecção pela destruição da Brucella ${ }^{14 .}$ Os derivados do leite, mormente os preparados do leite cru, se constituem em importante fonte de infecção ${ }^{6}$. Por estes motivos foram investigados também estes aspectos, relacionando-os com a procedência do leite, a duração do uso e a quantidade de ingestão diária de leite.

Embora a brucelose humana seja de dificil diagnóstico através da história clínica $\mathrm{e}$ do exame físico, foi decidido investigar alguns aspectos relacionados ao estado de saúde das pessoas estudadas que pudessem oferecer algum subsidio em relação à presença da doença.

$O$ uso indiscriminado de antibióticos de largo expectro pode mascarar o quadro de brucelose ${ }^{6}$, por isso, foi considerado que esta informação fosse também de importância.

Finalmente, como são os exames laboratoriais as unicas provas de certeza para o diagnóstico de brucelose, já que os sinais e sintomas da doença são inespecíficos $1 \%$, coletou-se sangue da população exposta ao risco para imunodiagnósticos.

Decidiu-se que duas provas seriam feitas; uma no sentido de se detectar os possiveis casos agudos e outra mais específica para as fases crônicas da doença *. Para diagnóstico da fase aguda foram feitas reaçōes de aglutinação lenta em tubo com antigeno de Brucella abortus; para o diagnóstico de doentes crônicos foram feitas reações de fixação de complemento para brucelose 1 . Todos os soros foram submetidos às duas provas.
As informaçōes foram coletadas através do preenchimento de formulário próprio, com previsão para codificação e transcrição em cartões IBM para processamento eletrônico dos dados.

As diferenças estatisticas encontradas foram testadas pelo método do qui quadrado:

\section{RESULTADOS}

\section{Descrição dos animais}

Dada a importância do sexo do animal na cadeia epidemiológica da bruceloses, na Tabela 1 é apresentada a distribuição do rebanho segundo sexo e resultado da reação de soroaglutinação para brucelose.

$\mathrm{Na}$ Tabela 2 estão apresentados os resultados da reação de soroaglutinação segundo a presença de lactação das vacas durante a época do levantamento.

\section{Populaçāo exposta ao risco}

Os consumidores do leite proveniente do rebanho em questão são apresentados na Tabela 3 , segundo sexo, cor e idade por grupo etário de acordo com a recomendação da Organização Mundial da Saúde '

A ocupação (ou profissão) reflete de alguma maneira as condições sócio-econômicas dos individuos, as quais interferem no estado sanitário e, portanto, nas condiçōes de saude de grupos populacionais. Com o objetivo de avaliar este aspecto, a população exposta é apresentada na Tabela 4 segundo a ocupação (ou profissão) e estado civil.

Alguns consumidores poderiam ter adquirido a doença em outras regióes onde residiam anteriormente, uma vez que $94,48 \%$ deles mencionaram o uso de leite "in natura" em alguma época da vida. Por outro lado, o tempo de uso do leite contaminado aumenta o risco de contrair a infeção, por isso são apresentados na Tabela 5 os consumidores segundo estas duas particularidades. 
Sotza. A. P. de et al. - Investigação da bruce lose em borinos e em consumidores humanos do leite, Rev. Saúde públ., S. Paulo. 11:238-47 1977.

\section{T A B E L A 1}

Rebanho bovino segundo sexo e resultado da reação de soroaglutinação para brucelose, município de Dumont, Estado de São Paulo.

\begin{tabular}{|c|c|c|c|c|c|c|}
\hline \multirow[b]{2}{*}{$\begin{array}{l}\text { Reação } \\
\text { p/ Brucelose }\end{array}$} & \multicolumn{2}{|c|}{ MACHO } & \multicolumn{2}{|c|}{ FEMEA } & \multicolumn{2}{|c|}{ TOTAL } \\
\hline & No & $\%$ & No & $\%$ & No & $\%$ \\
\hline Negativa & 2 & $(1,85)$ & 78 & $(72,22)$ & 80 & $(74,07)$ \\
\hline Suspeita & 1 & $(0,99)$ & 9 & $(8,33)$ & 10 & $(9,26)$ \\
\hline Positiva & - & & 18 & $(16,67)$ & 18 & $(16,67)$ \\
\hline TOTAL & 3 & $(2,78)$ & 105 & $(97,22)$ & 108 & $(100,00)$ \\
\hline
\end{tabular}

T A B E L A 2

Estado de lactação e resultado da reação de soroaglutinação para brucelose das vacas do rebanho bovino, municipio de Dumont. Estado de São Paulo

\begin{tabular}{|c|c|c|c|c|c|c|}
\hline \multirow[b]{2}{*}{$\begin{array}{l}\text { Reação } \\
\text { p/ Brucelose }\end{array}$} & \multicolumn{2}{|c|}{ NÃO LACTANTES } & \multicolumn{2}{|c|}{ EM LACTAÇÃO } & \multicolumn{2}{|c|}{ TOTAL } \\
\hline & Niy & $\%$ & No & $\%$ & $N^{\prime \prime}$ & $\%$ \\
\hline Negativa & 54 & $(51,43)$ & 24 & $(22,86)$ & 78 & $(74,29)$ \\
\hline Suspeita & 4 & $(3,81)$ & 5 & $(4,76)$ & 9 & $(8,57)$ \\
\hline Positiva & 15 & $(14,29)$ & 3 & $(2,86)$ & 18 & $(17,14)$ \\
\hline TOTAL & 73 & $(69,52)$ & 32 & $(30.48)$ & 105 & $(100,00)$ \\
\hline
\end{tabular}

T A B E L A 3

População exposta do risco segundo sexo, col e idade

\begin{tabular}{|c|c|c|c|c|c|c|c|}
\hline \multirow[b]{2}{*}{ Idade (anos) } & \multicolumn{3}{|c|}{ MASCULINO } & \multicolumn{3}{|c|}{ FEMININO } & \multirow{2}{*}{ rOTAI } \\
\hline & B & A & TOTAL & B & A & TOTAL & \\
\hline Até 1 & $\begin{array}{c}1 \\
(1.41)\end{array}$ & - & $\begin{array}{c}1 \\
(1.41)\end{array}$ & - & $\begin{array}{c}1 \\
(1.41)\end{array}$ & $\begin{array}{c}1 \\
(1.41)\end{array}$ & $\begin{array}{c}2 \\
(2.82)\end{array}$ \\
\hline $1-14$ & $\begin{array}{c}14 \\
(19.72)\end{array}$ & - & $\begin{array}{c}14 \\
(19,72)\end{array}$ & $\begin{array}{c}11 \\
(15.49)\end{array}$ & $\begin{array}{c}4 \\
(5.68)\end{array}$ & $\begin{array}{c}15 \\
(21.13)\end{array}$ & $\begin{array}{c}29 \\
(40.85)\end{array}$ \\
\hline $15-44$ & $\begin{array}{c}15 \\
(21,13)\end{array}$ & $\begin{array}{c}2 \\
(2,82)\end{array}$ & $\begin{array}{c}17 \\
(23,94)\end{array}$ & $\begin{array}{c}16 \\
(22,54)\end{array}$ & $\begin{array}{c}1 \\
(1,41)\end{array}$ & $\begin{array}{c}17 \\
(23,94)\end{array}$ & $\begin{array}{c}34 \\
(47,89)\end{array}$ \\
\hline $45-64$ & $\begin{array}{c}3 \\
(4.23)\end{array}$ & - & $\begin{array}{c}3 \\
(4,23)\end{array}$ & $\begin{array}{c}1 \\
(1.41)\end{array}$ & - & $\begin{array}{c}1 \\
(1,41)\end{array}$ & $\begin{array}{c}4 \\
(5.63)\end{array}$ \\
\hline 65 e $\rightarrow$ & $\begin{array}{c}1 \\
(1,41)\end{array}$ & - & $\begin{array}{c}1 \\
(1,41)\end{array}$ & $\begin{array}{c}1 \\
(1,41)\end{array}$ & - & $\begin{array}{c}1 \\
(1.41)\end{array}$ & $\begin{array}{c}2 \\
(2,82)\end{array}$ \\
\hline TOTAL & $\begin{array}{c}34 \\
(47.89)\end{array}$ & $\begin{array}{c}2 \\
(2.82)\end{array}$ & $\begin{array}{c}36 \\
(50.70)\end{array}$ & $\begin{array}{c}29 \\
(40,85)\end{array}$ & $\begin{array}{c}6 \\
(8,45)\end{array}$ & $\begin{array}{c}35 \\
(49.30)\end{array}$ & $\begin{array}{c}71 \\
(100,00)\end{array}$ \\
\hline
\end{tabular}

$\mathrm{B}=$ Branco

$A=$ Amarelo 
SOUZA, A. P. de et al. - Investigação da brucelose em bovinos e em consumidores humanos do leite, Rev. Saúde públ., S. Paulo, 11:238-47 1977.

\section{TA B E L A 4}

População exposta ao risco segundo estado civil e ocupação

\begin{tabular}{|c|c|c|c|c|c|}
\hline \multirow[b]{2}{*}{ Frofissão } & \multirow[t]{2}{*}{ Menor } & \multirow[t]{2}{*}{ Solteiro } & \multirow[t]{2}{*}{ Casado } & \multirow[t]{2}{*}{ Viuvo } & \multirow[t]{2}{*}{ TOTAI } \\
\hline & & & & & \\
\hline Menor & $\begin{array}{c}21 \\
(29,58)\end{array}$ & - & - & - & $\begin{array}{c}21 \\
(29,58)\end{array}$ \\
\hline Estudante & $\begin{array}{c}10 \\
(14,08)\end{array}$ & - & - & - & $\begin{array}{c}10 \\
(14,08)\end{array}$ \\
\hline Do lar & - & 一 & $\begin{array}{c}1: \\
(18,31)\end{array}$ & $\begin{array}{c}1 \\
(1.41)\end{array}$ & $\begin{array}{c}14 \\
(19,72)\end{array}$ \\
\hline Comerciário & - & $\begin{array}{c}4 \\
(5,63)\end{array}$ & $\begin{array}{c}6 \\
(8,45)\end{array}$ & - & $\begin{array}{c}10 \\
(14,08)\end{array}$ \\
\hline Professor & - & - & $\begin{array}{c}2 \\
(2,82)\end{array}$ & 一 & $\begin{array}{c}2 \\
(2,8 \div)\end{array}$ \\
\hline Motorista & - & - & $\begin{array}{c}4 \\
(5,63)\end{array}$ & - & $\begin{array}{c}4 \\
(5.6 \%)\end{array}$ \\
\hline Militar & - & - & $\begin{array}{c}3 \\
(4.23)\end{array}$ & - & $\begin{array}{c}3 \\
(4,23)\end{array}$ \\
\hline Agricultor & - & $\begin{array}{c}1 \\
(1,41)\end{array}$ & $\begin{array}{c}4 \\
(5.63)\end{array}$ & - & $\begin{array}{c}5 \\
(7,04)\end{array}$ \\
\hline Industriário & - & $\begin{array}{c}2 \\
(2,82)\end{array}$ & - & - & $\begin{array}{c}2 \\
(2,82)\end{array}$ \\
\hline TOTAL & $\begin{array}{c}31 \\
(43.66)\end{array}$ & $\begin{array}{c}7 \\
(9.86)\end{array}$ & $\begin{array}{c}32 \\
(45.07)\end{array}$ & $\begin{array}{c}1 \\
(1,41)\end{array}$ & $\begin{array}{c}71 \\
(100,00)\end{array}$ \\
\hline
\end{tabular}

\section{TABELA 5}

População exposta ao lisco segundo tempo de residência em Dumont, Estado de São Paulo e tempo de uso do leite contaminado

\begin{tabular}{|c|c|c|c|c|c|}
\hline Tempo & Não usa & até 1 ano & 1 a 4 anos & 5 a 9 anos & TOTAL \\
\hline Ate 1 ano & $\begin{array}{c}2 \\
(2,82)\end{array}$ & 一 & - & - & $\begin{array}{c}2 \\
(2,82)\end{array}$ \\
\hline $1-4$ & - & $\begin{array}{c}6 \\
(8.45)\end{array}$ & $\begin{array}{c}14 \\
(19,72)\end{array}$ & - & $\begin{array}{c}20 \\
(27,17)\end{array}$ \\
\hline $5-9$ & - & $\begin{array}{c}1 \\
(1,41)\end{array}$ & $\begin{array}{c}4 \\
(5,68)\end{array}$ & $\begin{array}{c}6 \\
(8,45)\end{array}$ & $\begin{array}{c}11 \\
(15,49)\end{array}$ \\
\hline $19-19$ & - & $\ldots$ & $\begin{array}{c}4 \\
(5.63)\end{array}$ & $\begin{array}{c}10 \\
(14,08)\end{array}$ & $\begin{array}{c}14 \\
(19,72)\end{array}$ \\
\hline $20-89$ & $\begin{array}{c}1 \\
(1.41)\end{array}$ & - & $\begin{array}{c}12 \\
(16.90)\end{array}$ & $\begin{array}{c}1 \\
(1.41)\end{array}$ & $\begin{array}{c}14 \\
(19.72)\end{array}$ \\
\hline $411-79$ & $\begin{array}{c}2 \\
(2,82)\end{array}$ & - & $\begin{array}{c}8 \\
(11,27)\end{array}$ & - & $\begin{array}{c}10 \\
(14,08)\end{array}$ \\
\hline TOTAL & $\begin{array}{c}5 \\
(7,(14)\end{array}$ & $\begin{array}{c}7 \\
(9,86)\end{array}$ & $\begin{array}{c}42 \\
(59.15)\end{array}$ & $\begin{array}{c}17 \\
(23.94)\end{array}$ & $\begin{array}{c}71 \\
(100,00)\end{array}$ \\
\hline
\end{tabular}


SouzA, A. P. de et al. - Investigação da brucelose em bovinos e em consumidores humanos do leite, Rev. Saúde públ., S. Paulo, 11:238-47 1977.

Na Tabela 6 é apresentada a distribuição segundo a quantidade média de ingestão diária do leite, bem como os tipos de leite usados, uma vez que algumas famílias utilizam-no de mais de uma fonte, o que pode interferir no aparecimento da doença.

As condições de saúde da população exposta, bem como o tratamento medicamentoso, em especial a antibioticoterapia, são apresentadas na Tabela 7 .

A distribuição de frequeência das principais morbidades especificas mencionadas pela população exposta ao risco é apresentada na Tabela 8.

É necessário salientar que a grande maio- ria dos consumidores - 95,45\% - revelou ferver criteriosamente o leite antes do uso; $1,52 \%$ da população fervia irregularmente o leite e $3,03 \%$, apenas, não fazia qualquer tratamento do leite antes do uso.

Os derivados do leite (manteiga, queijo, coalhada, etc.) não são utilizados pela população.

Dos 71 consumidores humanos do leite, foram colhidas amostras de sangue para as reações sorológicas de $69 \quad(97,18 \%)$ tendo sido excluidas duas crianças com idade inferior a 3 meses e que só usavam leite materno ou leite em pó. Das 69 amostras colhidas nenhuma foi positiva sorologicamente.

T A B E L A 6

População exposta ao risco segundo tipo de leite ingerido e a quantidade de ingestão diária média

\begin{tabular}{|c|c|c|c|c|c|}
\hline Tipo $\begin{array}{c}\text { Quantidade de } \\
\text { deite usado }\end{array}$ & Não houve & até $300 \mathrm{ml}$ & $301-600$ & $600-1000$ & TOTAL \\
\hline Nenhum & $\begin{array}{c}5 \\
(7,04)\end{array}$ & - & - & - & $\begin{array}{c}5 \\
(7,04)\end{array}$ \\
\hline "in natura" & - & $\begin{array}{c}27 \\
(29.58)\end{array}$ & $\begin{array}{c}2 \\
(2.82)\end{array}$ & $\begin{array}{c}2 \\
(2,82)\end{array}$ & $\begin{array}{c}31 \\
(43,66)\end{array}$ \\
\hline pasteurizado & - & $\begin{array}{c}1 \\
(1.41)\end{array}$ & - & - & $\begin{array}{c}1 \\
(1,41)\end{array}$ \\
\hline enı pó & - & - & $\begin{array}{c}1 \\
(1.41)\end{array}$ & - & $\begin{array}{c}1 \\
(1,41)\end{array}$ \\
\hline $\begin{array}{l}\text { in natura }+ \\
\text { pasteurizado }\end{array}$ & - & $\begin{array}{c}5 \\
(7,04)\end{array}$ & $\begin{array}{c}2 \\
(2,82)\end{array}$ & - & $\begin{array}{c}7 \\
(9,86)\end{array}$ \\
\hline $\begin{array}{l}\text { in natura }+ \\
\text { em pó }\end{array}$ & - & - & $\begin{array}{c}3 \\
(4,23)\end{array}$ & - & $\begin{array}{c}3 \\
(4,23)\end{array}$ \\
\hline $\begin{array}{l}\text { in natura }+ \\
\text { materno }\end{array}$ & - & $\begin{array}{c}3 \\
(4,23)\end{array}$ & $\begin{array}{c}4 \\
(5,63)\end{array}$ & $\begin{array}{c}5 \\
(7.04)\end{array}$ & $\begin{array}{c}12 \\
(16,90)\end{array}$ \\
\hline $\begin{array}{l}\text { em po }+ \\
\text { materno }\end{array}$ & - & - & - & $\begin{array}{c}1 \\
(1.41)\end{array}$ & $\begin{array}{c}1 \\
(1.41)\end{array}$ \\
\hline $\begin{array}{l}\text { in natura }+ \text { pasteu- } \\
\text { rizado }+ \text { em pó }\end{array}$ & - & - & $\begin{array}{c}1 \\
(1.41)\end{array}$ & $\begin{array}{c}1 \\
(1,41)\end{array}$ & $\begin{array}{c}2 \\
(2.82)\end{array}$ \\
\hline $\begin{array}{l}\text { in natura + pasteu- } \\
\text { rizado }+ \text { materno }\end{array}$ & - & - & - & $\begin{array}{c}1 \\
(1,41)\end{array}$ & $\begin{array}{c}1 \\
(1,41)\end{array}$ \\
\hline $\begin{array}{l}\text { in natura }+ \text { em pó } \\
+ \text { materno }\end{array}$ & - & $\begin{array}{c}2 \\
(2,82)\end{array}$ & $\begin{array}{c}1 \\
(1,41)\end{array}$ & $\begin{array}{c}1 \\
(1,41)\end{array}$ & $\begin{array}{c}4 \\
(5,63)\end{array}$ \\
\hline $\begin{array}{l}\text { in natura }+ \text { pasteu- } \\
\text { rizado }+ \text { pó }+ \text { materno }\end{array}$ & - & $\begin{array}{l}2 \\
(2,82)\end{array}$ & - & - & $\begin{array}{c}2 \\
(2,82)\end{array}$ \\
\hline $\begin{array}{l}\text { in natura + pasteu- } \\
\text { rizado }+ \text { pó }+ \\
\text { outro }\end{array}$ & - & - & - & $\begin{array}{c}1 \\
(1,41)\end{array}$ & $\begin{array}{c}1 \\
(1,41,\end{array}$ \\
\hline TOTAL & $\begin{array}{c}5 \\
(7,04)\end{array}$ & $\begin{array}{c}40 \\
(56,34)\end{array}$ & $\begin{array}{c}14 \\
(19,72)\end{array}$ & $\begin{array}{c}12 \\
(16.90)\end{array}$ & $\begin{array}{c}71 \\
(100,00)\end{array}$ \\
\hline
\end{tabular}


SoUZA, A. P. de et al. - Investigação da brucelose em bovinos e em consumidores humanos do leite. Rev. Saúde públ., S. Paulo, 11:238-47 1977.

TABELA 7

População exposta ao risco segundo as condições de saúde e tratamento medicamentoso no periodo de uso do leite

\begin{tabular}{|c|c|c|c|c|c|}
\hline Condições & Nenhum & Antibiótico & Outro & Não sabe & TOTAI \\
\hline Boas & $\begin{array}{c}39 \\
(54,93)\end{array}$ & $\begin{array}{c}7 \\
(9,86)\end{array}$ & $\begin{array}{c}1 \\
(1.41)\end{array}$ & $\begin{array}{c}1 \\
(1,41)\end{array}$ & $\begin{array}{c}48 \\
(67,61)\end{array}$ \\
\hline Doença aguda & $\begin{array}{c}4 \\
(5,63)\end{array}$ & $\begin{array}{c}5 \\
(7,04)\end{array}$ & $\begin{array}{c}3 \\
(4,28)\end{array}$ & - & $\begin{array}{c}12 \\
(16,90)\end{array}$ \\
\hline Doença crônica & $\begin{array}{c}2 \\
(2,82)\end{array}$ & $\begin{array}{c}4 \\
(5,63)\end{array}$ & $\begin{array}{c}5 \\
(7,04)\end{array}$ & - & $\begin{array}{c}11 \\
(15,49)\end{array}$ \\
\hline TOTAL & $\begin{array}{c}45 \\
(63,38)\end{array}$ & $\begin{array}{c}16 \\
(22,54)\end{array}$ & $\begin{array}{c}9 \\
(12,68)\end{array}$ & $\begin{array}{c}1 \\
(1,41)\end{array}$ & $\begin{array}{c}71 \\
(100,00)\end{array}$ \\
\hline
\end{tabular}

T A B E L A 8

População exposta ao risco segundo as morbidades referidas

\begin{tabular}{l|c}
\hline Morbidade & Freqüencia \\
\hline Sem queixas & 46 \\
Amigdalite & 1 \\
Artralgia & 2 \\
Bronquite & 2 \\
Cardiopatia congênita & 1 \\
Cefaléia & 2 \\
Colecistopatia & 1 \\
Epilepsia & 2 \\
Febre reumática & 1 \\
Hipertensão & 1 \\
Infecção intest. & 1 \\
Surdez & 1 \\
Nódulo mamário & 1 \\
Psicopatia & 1 \\
Resfriado & 2 \\
Reumatismo & 1 \\
Rinite & 1 \\
Ũlcera-péptica & 3 \\
Outros & 1 \\
Total & 71 \\
\hline & \\
\hline
\end{tabular}

\section{I S CUSSAO}

Segundo Hobson ${ }^{6}$ a taxa de infecção por brucelose entre os bovinos varia de 1 a $30 \%$ nas diversas regiōes do mundo. No rebanho estudado esta percentagem foi de $16,67 \%$ de positivos e $9,26 \%$ de suspeitos, perfazendo um total de $25,9 \%$ (Tabela 1) que se aproxima do limite superior acima mencionado.

De acordo com Valente \& Amaral ${ }^{13}$, na região de Ribeirão Preto, da qual o município de Dumont faz parte, existia $9,7 \%$ de animais com sorologia positiva e $7,1 \% \mathrm{com}$ sorologia duvidosa (suspeitos) para brucelose no período de 1965 a 1967. Estes dados sugerem maior prevalência da doença no rebanho estudado em 1975 do que aquela da região. Pressupondo independência entre estas populações de animais, em virtude da defasagem cronológica, as diferenças mostraram-se estatisticamente significantes pelo teste de qui quadrado. $(\mathrm{p}=0,02090)$.

Analisando-se a Tabela 2 , observa-se que na época do levantamento, $9,38 \%$ das vacas em lactação apresentaram sorologia positiva para brucelose e $15,63 \%$ eram suspeitas perfazendo um total de $25 \%$. Entre as não lactantes esta percentagem era maior $26,03 \%$ - principalmente para aquelas positivas $20,55 \%$. Como o leite foi fornecido durante um longo período (9 anos ou mais - Tabela 5), seguramente houve participação da maioria das vacas brucélicas na produção do leite fornecido à população humana em estudo. Isto leva a concluir, com certa segurança, que o forne- 
SoUzA, A. P. de et al. - Investigação da brucelose em bovinos e em consumidores humanos do leite. Rev. Saúde públ., S. Paulo, 11:238-47 1977.

cimento de leite contaminado ocorreu por largo espaço de tempo, oferecendo condições da transmissão da doença aos consumidores, caso outras medidas não tenham sido tomadas.

A análise da Tabela 3 demonstra inexistência de diferença quantitativa relacionada ao sexo dos indivíduos expostos ao consumo de leite, e que a maioria das pessoas é menor de 45 anos, embora existam pessoas de todos os grupos etários.

Os resultados dos exames laboratoriais para diagnóstico sorológico da brucelose (fixação de complemento e soroaglutinação) foram negativos para todas as pessoas examinadas. Respeitando-se nas limitações biológicas de qualquer reação sorológica, pode-se concluir que esta população mesmo residindo por longo tempo na área $e$ fazendo uso constante do leite contaminado (Tabela 5) não adquiriu brucelose. Corroborando este fato, embora a história clínica da brucelose seja inespecífica, o levantamento das morbidades especificas referidas pela população estudada não revelou um só caso que pudesse levar a suspeita da brucelose, apesar de $16,90 \%$ das pessoas apresentarem algum tipo de doença aguda $\mathrm{e}$ $15,49 \%$ apresentarem doença crônica, como pode ser observado na Tabela 7 .

$\mathrm{Na}$ Tabela 8 são apresentadas as morbidades específicas referidas pelos entrevistados e observa-se que algumas queixas como artralgia ( 2 casos), reumatismo (1 caso), etc. embora pudessem levar a suspeita de brucelose, longe estão de permitir o diagnóstico da doença diante dos resultados sorológicos negativos.

Alguns achados podem justificar estes resultados. Em primeiro lugar, 95,45\% dos consumidores fervem criteriosamente o leite antes do uso. Como se sabe, no leite a Brucella é prontamente destruída pela manutenção em alta temperatura $\left(100^{\circ} \mathrm{C}\right)^{14}$; além disto apenas $43,66 \%$ da população exposta usa somente o leite "in natura", os demais fazem uso de outras apresentaçōes lacteas como: leite pasteurizado, leite em pó, etc. (Tabela 6).

A disseminação do uso de antibióticos tem concorrido para o bloqueio da multiplicação da Brucella e do desenvolvimento de titulos significativos de anticorpos ${ }^{\circ}$. Como se observa na Tabela $7,22,55 \%$ dos entrevistados fizeram uso de antibiótico no período de exposição ao risco da doença, mesmo aqueles que se encontravam em boas condições de saúde $(9,86 \%)$.

Os derivados do leite cru são considerados como vias de extrema importância na transmissāo da infecção (1 e 10), entretanto, a população exposta não tinha o hábito de utilizar estes derivados do leite contaminado.

Finalmente, ao se analisar a Tabela 4 pode-se observar que os membros da população possuem profissões relativamente diferenciadas, que por si só podem indicar maiores precauções em relação a transmissão da Brucelose.

\section{CONCLUSOES}

1. O rebanho bovino estudado apresentou, à soroaglutinação para brucelose, uma percentagem de $16,67 \%$ de positivos e $9,26 \%$ de suspeitos, perfazendo um total de $25,9 \%$.

2. Das vacas em lactação, $9,38 \%$ apresentaram sorologia positiva para brucelose, enquanto $15,63 \%$ foram classificadas como suspeitas. Entre as não lactantes a percentagem de positivas foi de $20,55 \%$ e de suspeitas de $5,48 \%$.

3. O leite do rebanho foi fornecido durante um longo período ( 9 anos ou mais), havendo, assim, participação da maioria das vacas na produção do leite fornecido à população humana estudada. 
SoUZA, A. P. de et al. - Investigação da brucelose em bovinos e em consumidores humanos do leite. Rer. Saúde públ., S. Paulo, 11:238-47 1977.

4. A população mesmo residindo por longo tempo na área e fazendo uso constante do leite não adquiriu brucelose. (Os exames laboratoriais para diagnóstico sorológico da brucelose humana foram todos negativos).

5. Uma das explicações para o não aparecimento da doença entre os consumidores é a fervura criteriosa do leite antes do uso. $(94,45 \%$ das pessoas informaran tomar esta providnêcia).

\section{A GR A D E I M ENTOS}

Ao Prof. Dr. José Oliveira de Almeida, Professor Catedrático do Departamento de Microbiologia, Imunologia e Parasitologia da Faculdade de Medicina de Ribeirão Preto - USP, pelos resultados dos exames de suroaglutinação e fixação de complemento, e ao Sr. Oswaldo de Campos Borelli pelo auxílio no trabalho de campo junto à população.

RSPU-B/355

SouzA, A. P. de et al. [Investigation on Brucellosis in cattle and human consumers of milk.] Rev. Saúde públ., S. Paulo, $11: 238-47,1977$.

ABSTRACT: The use of "in natura" milk by some communities became a serions: problem in Public Health. A revision on the Bracellosis situation in bovine herds in the county of Ribeirão Preto was understaken. From the detection of Bracellic milk producer bovine herd, the human consumers of the suspected milk were investigated according to some characteristics, such as: sex, colour, occupation, marital status, time residence in the area, time of use of the suspected milk, kind of ingested milk, average amount of daily milk ingestion, health conditions, use of drugs and referred morbidity at the time of the study. Serological diagnostic tests for Brucellosis in the milk producer bovine herd and in the milk were also performed. It could be stressed that about 8 per cent of the cows in lactation by the time of the survey were Bracellic. Among the consumers no positive serological test were found for Bracellosis. Is was concluded that: despite the importance of Brucellosis as a zoonosis, it persists endemic in our breeding environment; the boiling of the milk is an efficient measure. It should be recommended in areas where "in natura" milk is consumed.

Uniterms: Brucellosis. Bracellosis, bovine. Milk "in natura".

\section{REFERENCIAS BIBLIOGRAFICAS}

1. ALMEIDA, J. O. Standartized quantitative complement - fixation test. Immunochem. and Immunol., 2:248494, 1972 .

2. AMERICAN PUBLIC HEALTH ASSOCIATION. Profilaxia das doenças trans-

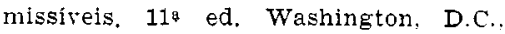
Organização Panamericana da Saúde. 1973. (OPAS - Publ. cient., 268).

3 ARMITAGE, P. Further analysis of qualitative data. In: Statistical methods in medical research. Oxford,
Blackwell Scientific Publications, 1971. p. 362-75.

4. BUSCH, L. A. \& PARKER, R. L. Brucelosis in the United States. J. infect Dis., 125:289-94, 1972, Apud ZOONOSIS, Ramos Mejia, 14:156, 1972.

5. COMITE MIXTO FAO/OMS DE EXPERTOS EN BRUCELOSIS, Ginebra, 1970. Informe: $\breve{5}^{\varphi}$. Ginebra, 1971, (OMS Ser. Inf. teen., 464).

6. HOBSON, W. The theory and practice of public health. 4th Ed. London, Oxford University Press, 1975. 
SoUzA, A. P. de et al. - Investigação da brucelose em bovinos e em consumidores humanos do leite. Rev. Saúde públ., S. Paulo, 11:238-47 1977.

7. MANUAL đa Classificação estatística Internacional de Doenças, Lesões e Causas de Obito. Revisão 1965. Washington, D.C., Organização Panamericana da Saúde, 1969. 2v. (OPAS - Publ. cient., $190)$.

8. RUNNELLS, $\mathrm{R}$. A. et al. Principios de patologia veterinaria. Mexico, Comp. Ed. Continental, 1968.

9. SANTOS, J. A. \& MELLO, M. R. Diagnóstico médico-veterinârio (colheita de materiat). São Paulo, Nobel, 1974. p. 87-8.

10. SATHLER, I. Brucelose: a bomba relógio. Boletim do Campo, 37:2-5, 1974 .

11. SHILF, E. A. Progress report on cooperative state federal brucellosis erra- dication program. Washington, D. C. U. S. Department of Agriculture, 1968.

12. SPINK. W, W. Brucelose (Febre ondulante). In: HARRISON, T. H. Medicina interna. 4 ed. Rio de Janeiro. Guanabara - Koogan, 1968. p. 1706-9.

13. VALENTE, F. de A. T. \& AMARAL, L. B. Brucelose bovina no Estado de São Paulo. Biologico, 35/36, 1969/70. Apud ZOONOSIS, Ramos Mejia, 13:171-2. 1971.

14. WILSON, G. S. \& MILES, A. A. Topley and Wilson's principles of bacteriology and immunity. 5th ed. London. Edward Arnold, 1964. v. 1, p. 992-1018.

Recebido para publicaçāo em 8/11/1976 Aprovado para publicasāo em 1\%/12/1976 